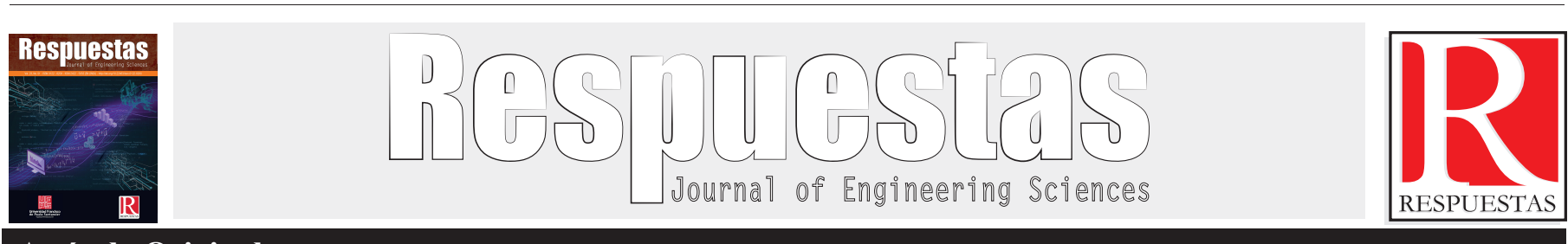

Artículo Original

https://doi.org/10.22463/0122820X.1504

\title{
Análisis de metodologías para la evaluación de cadenas de valor: caso del aguacate en Norte de Santander
}

\author{
Analysis of methodologies for the assessment of value chains: avocado case in Norte de Santander \\ Eduardo Felipe Vásquez-Barajas, Liliana Marcela Bastos-Osorio. \\ Profesional en Comercio Internacional, eduardofelipevb@ufps.edu.co, orcid.org/0000-0001-6871-9140, Universidad Francisco de Paula Santander, Cúcuta, Colombia \\ Magister en Gerencia de Empresas, lilianamarcebo@ufps.edu.co, orcid.org/0000-0001-6315-4224, Universidad Francisco de Paula Santander, Cúcuta, Colombia.
}

Cómo citar: E.F. Vásquez-Barajas, L.M. Bastos-Osorio, “Análisis de metodologías para la evaluación de cadenas de valor: caso del aguacate en Norte de Santander", Respuestas, vol. 23, no. S1, pp. 66-69, 2018. https://doi.org/10.22463/0122820X.1504

Recibido: Febrero 12, 2018; Aprobado: Julio 25, 2018.

\begin{tabular}{ll}
\hline RESUMEN \\
\hline Palabras Clave: & El presente artículo tiene como objetivo analizar metodologías de evaluación de cadenas de valor que luego pueden aplicarse a la cadena \\
Aguacate & de valor del aguacate en Norte de Santander. En cuanto a la metodología, el tipo de investigación es cuantitativo, con método de investi- \\
Cadena de valor & gación deductivo y tipo de estudio descriptivo sobre el aguacate en Norte de Santander y metodologías para evaluación de cadena de \\
Evaluación & valor. Sobre los resultados, el aguacate no registra como una de las principales frutas cultivadas en Norte de Santander pero sí es una \\
Internacionalización & con las de mayor potencial de crecimiento en área de cultivo y potencial internacional debido a la demanda internacional de principales \\
Norte de Santander & variedades como "Hass". Por lo tanto, la cadena de valor bajo el modelo de Michael Porter se constituye en una herramienta clave para \\
& diagnosticar la línea productiva de aguacate en Norte de Santander. Lo anterior conlleva a analizar metodologías existentes para evaluar \\
& cadenas de valor y sus interacciones entre las actividades primarias y las actividades de apoyo así como sus eslabones y factores \\
& externos identificando el grado de generación de valor que conlleve a la competitividad y potencial internacionalización. Por lo tanto, \\
& se concluye como pertinente el análisis de metodologías existentes para la evaluación de cadenas de valor que sirvan como antecedente \\
& para la construcción de una nueva metodología o puedan ser aplicadas en la evaluación de la cadena de valor de aguacate de Norte de \\
& Santander con el fin de identificar a través de las actividad principales y de apoyo las ventajas competitivas y factores por mejorar, \\
& tomando los resultados como base para una futura internacionalización del aguacate de Norte de Santander.
\end{tabular}
tomando los resultados como base para una futura internacionalización del aguacate de Norte de Santander.

$\begin{array}{ll}\text { Keywords: } & \begin{array}{l}\text { The objective of this article is to analyze some methodologies for assessing value chains that can be applied then to the value chain of } \\ \text { avocado in Norte de Santander. Regarding the article's methodology, the research type is quantitative, a deductive research method and } \\ \text { Avocado }\end{array} \\ \text { Assessment } & \text { a type of descriptive study on avocado in Norte de Santander and some methodologies for the value chain assessment. As per the results, } \\ \text { the avocado is not one of the main fruits grown in Norte de Santander but it is one with the greatest growth potential in the area of } \\ \text { Norte de Santander } \\ \text { cultivation and international potential due to the international demand of main varieties such as "Hass". Therefore, the value chain in } \\ \text { accordance with the Michael Porter's model constitutes a key tool to diagnose the productive line of avocado in Norte de Santander. } \\ \text { This involves analyzing existing methodologies to assess value chains and their interactions between primary activities and support } \\ \text { activities as well as their links and external factors identifying the level of value generation that leads to competitiveness and a potential } \\ \text { internationalization. Thus, the analysis of existing methodologies for assessing value chains that serve as a precedent for the construc- } \\ \text { tion of a new methodology or can be applied in the assessment of the value chain of avocado in Norte de Santander is concluded as } \\ \text { pertinent in order to identify through the main activities and support the competitive advantages and factors to be improved, taking the } \\ \text { results as a basis for a future internationalization of the avocado from Norte de Santander. }\end{array}$

\section{Introducción}

El aguacate es una de las frutas que cuenta con mayor potencial de crecimiento a nivel nacional, motivado por la demanda actual y potencial en el mercado nacional y principalmente en el internacional. En el caso particular del aguacate en Norte de Santander, no es la principal fruta producida ni cuenta con una alta producción pero cumple con lo expuesto anteriormente sobre el potencial de crecimiento. Por lo tanto, se determina la necesidad del diseño de su cadena de valor partiendo de lo establecido por Porter [1] como referente teórico de esta herramienta de diagnóstico de sus actividades principales y de apoyo para la generación de valor.
La determinación de la cadena también conlleva a la necesidad de evaluarla con el objetivo de identificar las actividades y eslabones internos y externos donde se identifiquen ventajas diferenciales así como las actividades por fortalecer.

El presente artículo tiene por objetivo analizar dos metodologías de evaluación de cadenas de valor que pueden ser empleadas en el caso particular de la cadena de valor del aguacate en Norte de Santander. De este modo, el artículo presenta dentro de sus resultados la situación actual del aguacate en Norte de Santander, su cadena de valor y las metodologías a tener en cuenta para la evaluación de la cadena de valor. 


\section{Materiales y métodos}

Respecto a la metodología de investigación, el tipo de investigación es cuantitativo, con método de investigación deductivo y tipo de estudio descriptivo. Además, se realizaron búsquedas en español e inglés en bases de datos y repositorios como Science Direct, Redalyc y Dialnet para encontrar metodologías de evaluación de cadenas de valor a analizar e información sobre el aguacate, así mismo, se tuvo en cuenta la Agenda Regional de Competitividad de Norte de Santander y estadísticas gubernamentales a través de Asohofrucol y el portal Agronet del Ministerio de Agricultura y Desarrollo Rural.

\section{Resultados y Análisis}

El aguacate de Norte de Santander registró en el 2014 unas 213 hectáreas de las cuales se obtuvo una producción de 2.411,40 toneladas con un rendimiento de 11.32 ton/h, según lo reportado por Agronet. Además, Asohofrucol actualmente trabaja con los cultivadores apoyándolos en la obtención del certificado de buenas prácticas agrícolas y en aumentar las hectáreas en el año 2020 a 800 hectáreas, destinando 300 hectáreas para la variedad "Hass", la cual tiene un alto potencial de exportación por la alta demanda de consumo internacional. Otras variedades de importancia (ver Figura 1) en el departamento para comercialización e internacionalización pueden ser "Lorena" y "Choquette".

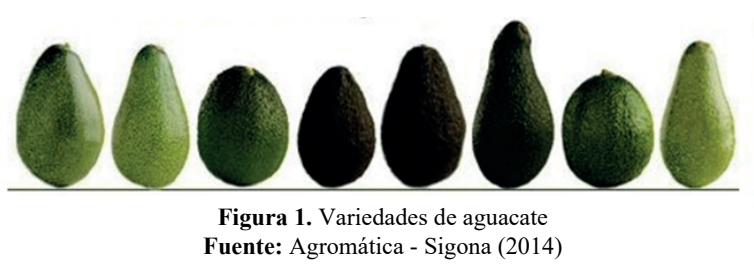

Según, Asohofrucol seccional Norte de Santander, municipios como Toledo, Labateca y Bochalema presentan producción de variedad "Hass" mientras que municipios como El Carmen, Convención, Bochalema y La Esperanza concentran una mayor producción de otras variedades verdes de aguacate como "Lorena".

Aun así, el aguacate de Norte de Santander requiere fortalecerse en la agregación de valor, la aplicación técnica y tecnológica, el cumplimiento de normas sanitarias y certificaciones exigidas para una internacionalización correcta [2].

Por lo tanto, según Rodríguez \& Mora [3] la internacionalización ha conllevado a que las economías nacional y regional busquen una mayor participación en los mercados internacionales más dinámicos, planteando un escenario global con mayor interdependendencia. Por otra parte, el interés por la comercialización internacional del aguacate se debe a los buenos precios internacionales, una alta demanda y una tasa de cambio Dólar Estadounidense / Peso Colombiano favorable para los exportadores, pues como según lo comenta Morales, Rojas \& Velázquez [4]. una pérdida de valor de la divisa nacional permite que las exportaciones de las empresas nacionales aumenten, produciendo una ganancia cambiaria al momento de convertir las divisas recibidas del extranjero a moneda nacional.

\section{Cadena de valor del aguacate en Norte de Santander}

La cadena de valor del aguacate se elabora con la identificación de las actividades primarias y de apoyo propuesta por Porter [1]:

- Actividades primarias: Logística de entrada, operaciones, logística de salida, marketing y ventas, servicio.

- Actividades de apoyo: Infraestructura, manejo de recursos humanos, desarrollo de tecnología, adquisiciones de soporte.

La cadena de valor permite identificar y analizar fuentes de la ventaja competitiva y diferenciación en las actividades primarias y de apoyo, apuntando a la generación de valor o margen. Además, como herramienta de diagnóstico se puede determinar dónde se deberían disminuir costos u optimizar beneficios, mejorar el nivel de comunicación, propiciar la transferencia tecnológica e identificar la gobernanza. [5] Además, se resalta la importancia del análisis de la cadena de valor para impactar positivamente la competitividad y disminuir los cuellos de botella [6].

Dentro de los actores que interaccionan en la cadena de valor están las asociaciones de cultivadores de aguacate en el departamento, la Asociación Hortofrutícola de Colombia (Asohofrucol), ICA, Corpoica el naciente Comité Regional de la Cadena del Aguacate de Norte de Santander, clientes, comercializadores, proveedores, gobierno y universidades.

En esta cadena se podrían identificar como actividades principatles fuertes la logística de entrada y operaciones (cultivo), siendo esta última soportada positivamente por el desarrollo de tecnología y sus actores para poder fortalecer y aumentar las operaciones (cultivo); en cuanto a infraestructura organizacional y manejo de recursos humanos se ha venido mejorando como actividades soporte.

Por otra parte, las actividades primarias a fortalecer serían la logística de salida o distribución que se realiza a nivel local y con cuellos de botella para garantizar un suministro, marketing y ventas así como servicios (seguimiento al clientes) requieren recibir atención en la cadena dado que prácticamente no han sido desarrolladas de manera estable; además, las adquisiciones de soporte también deben fortalecerse a través de facilidad en acceso al financiamiento y dotación de herramientas técnicas y empresariales para una mejor profesionalización del negocio.

\section{Metodologías para la evaluación de una cadena de valor:}

Metodología de evaluación por factor de integración: De acuerdo con De la Hoz, Fontalvo \& Morelos [7] en su investigación sobre un "Modelo de evaluación de cadenas de suministro en el sector de confecciones de Barranquilla", se detallan a continuación 
los factores claves en la integración de una cadena de suministro organizacional:

- Integración del cliente: Es un factor clave que permite a las empresas construir relaciones duraderas con clientes seleccionados. Dicha integración está determinada por seis elementos: Segmentación, importancia, agilidad, flexibilidad, información compartida y colaboración. [7]

- Integración de operaciones internas: Necesita de un compromiso de efectividad en los procesos y un esfuerzo coordinado para incrementar el desempeño y reducir los costos, lo anterior conlleva a mejorar la competitividad. Los elementos que la determinan son: Unificación interfuncional, estandarización, simplificación, conformidad, adaptación estructural, administración de información, comunicación interna y evaluación funcional. [7]

- Integración de proveedores de materiales y servicios: Tiene como fin dinamizar y coordinar los flujos internos y externos a través de una integración vertical. Puede conllevar a comprometer recursos adicionales de tiempo y capital. Los cinco elementos determinantes de dicha integración son: La alineación estratégica, la fusión operacional, las relaciones financieras, la administración de proveedores y compartir información [7].

Con los anteriores factores de integración se utiliza una escala de Likert de cinco puntos, donde 1 es total desacuerdo y 5 es total acuerdo) con un punto intermedio de 3 de ni en acuerdo ni en desacuerdo. [7].

La medición anterior permitiría evaluar el nivel de integración de la cadena de valor con sus proveedores (logística de entrada), su integración interna (operaciones y actividades de soporte) y la integración con sus clientes (actividades primarias de logística de salida, marketing y ventas, servicio).

Metodología de evaluación por análisis FODA y ponderación de actividades por categorías: Según Arce \& Calves en su proyecto "La evaluación de las cadenas de valor como estrategia para la competitividad de las Pymes" [8], se establece la siguiente metodología para la evaluación de una cadena de valor:

Se inicia con un análisis FODA de los aspectos externos e internos de la cadena de valor para obtener un diagnóstico detallado de las fortalezas, oportunidades, debilidades y amenazas para luego pasar a proponer las debidas estrategias. Luego se pasa a desarrollar una matriz de valor y sus ponderaciones sobre las actividades por categorías de la cadena de valor. La presente metodología se sustenta en el concepto de que la cadena de valor otorga categorías a las actividades que producen valor añadido en una organización y en base a sus componentes se elabora la matriz de valor. [8].
Los elementos de la matriz de valor son las mismas actividades principales y de apoyo que conforman la cadena de valor; en la columna irán las actividades principales mientras que en la fila irán las actividades de apoyo agregando dentro de las actividades de apoyo los conocimientos como actividad adicional, lo anterior se puede observar en la Tabla I, creada por los autores Pérez \& Calves [9] y citada por Arce \& Calves. [8].

Las categorías deben ponderarse para ser de utilidad en la toma de decisiones y en la identificación de puntos fuertes y los puntos por mejorar. La manera de ponderar consiste en asignar una calificación del 1 al 5 a cada una de las categorías definidas, siendo 1 el nivel más bajo y 5 el nivel más alto. [8].

Tabla I: Modelo de ponderación de actividades por categoria de codena de valor

\begin{tabular}{|c|c|c|c|c|c|}
\hline & $\begin{array}{l}\text { Infraestructura } \\
\text { de la empresa }\end{array}$ & $\begin{array}{l}\text { Recursos } \\
\text { Humanos } \\
\end{array}$ & \begin{tabular}{|l|} 
Desarrollo \\
tecnológico \\
\end{tabular} & Abasteciomientos & Conocimientos \\
\hline \multicolumn{6}{|l|}{$\begin{array}{l}\text { Logística } \\
\text { interna }\end{array}$} \\
\hline \multicolumn{6}{|l|}{ Operaciones } \\
\hline \multicolumn{6}{|l|}{$\begin{array}{c}\text { Logística } \\
\text { externa }\end{array}$} \\
\hline \multicolumn{6}{|l|}{$\begin{array}{l}\text { Marketing y } \\
\text { ventas }\end{array}$} \\
\hline Servicios & & & & & \\
\hline
\end{tabular}

Fuente: Pérez \& Calves (2008) citado por Arce \& Calves (2008)

La ponderación se da según el grado determinado en que una actividad principal está siendo fundamentada por una actividad de apoyo. Posteriormente, las categorías con las puntuaciones más bajas requerirían de acciones prioritarias de mejora [8].

\section{Conclusiones}

Por lo tanto, se concluye como pertinente el análisis de metodologías existentes para una adecuada evaluación de la cadena de valor que sirvan como antecedente para la construcción de una nueva metodología o puedan ser aplicadas en la evaluación de la cadena de valor de aguacate de Norte de Santander con el fin de identificar las actividad principales y de apoyo más fuertes y más débiles que conlleven a ventajas competitivas y factores por mejorar, tomando los resultados como base para un aumento de la productividad del cultivo y una futura internacionalización del aguacate de Norte de Santander en sus diversas variedades, como "Hass" y otras variedades como "Lorena" y "Choquette".

\section{Referencias}

[1] M. Porter. Ventaja competitiva. Quinta reimpresión. México: CECSA, 2006.

[2] Departamento Nacional de Planeación (DNP). Agenda interna para la productividad y la competitividad: Documento 
regional Norte de Santander, 2007. Disponible en: http://www.incoder.gov.co/documentos/Estrategia\%20d $\quad$ e $\quad \% \quad 2 \quad 0 \quad$ D $\quad$ e $\quad \begin{array}{lllll}\text { s } & \text { a } & -\end{array}$ rrollo\%20Rural/Pertiles\%20Territoriales/ADR\%20Sur\%20de $1 \% 20$ Cesar/Otra $\% 20$ Informacion/Agenda $\% 20$ interna $\% 20$ Nort e\%20de\%20Santander.pdf

[3] J. E. Rodríguez, A. Mora, "Diagnóstico de la logística del carbón en Norte de Santander", Respuestas, vol. 20, no. 1, pp. 30-37, 2015.

[4] J. A. Morales-Castro, M. Rojas-Ortega y G. Velázquez-Valadez, "Resultados cambiarios y su relación con el grado de internacionalización de las empresas de la Bolsa Mexicana de Valores", Respuestas, vol. 22, no. 1, pp. 59-74, 2017

[5] Y. Peña, P. Nieto y F. Díaz. Cadenas de valor: un enfoque para las agrocadenas. Equidad y Desarrollo, 0 (9), 77-85, 2008. DOI: http://dx.doi.org/10.19052/ed.279

[6] J. Chávez. Cadena de valor, estrategias genéricas y competitividad: el caso de los productores de café orgánico del municipio de Tanetze de Zaragoza, Oaxaca. México, 2012. Disponible en: http://www.eumed.net/libros-gratis/2013b/1345/tipo-investigacion.html

[7] E. De la Hoz, T. Fontalvo y J. Morelos. Modelo de evaluación de cadenas de suministro en el sector de confecciones de Barranquilla. Revista INGENIARE Universidad Libre Barranquilla, Año 6, No. 11, pp. 79-92, 2011

[8] B. Arce y S. Calves. La evaluación de las cadenas de valor como estrategia para la competitividad de las Pymes. Revista electrónica de ciencias sociales Tecsistecatl, ISSN 1886-8452 No. 5, diciembre, 2008.

[9] O. Pérez y S. Calves. Ponderación de las cadenas de valor. Curso a empresarios de la UNECA. Cuba, 2008. 